\title{
TENDENCIAS DEL HUMOR ÉTNICO: UN ANÁLISIS DE CHISTES CONTEMPORÁNEOS EN LENGUA RUSA
}

\author{
GUILLEM CASTAÑAR RUBIO \\ Universidad de Barcelona \\ gcastanar@gmail.com
}

\section{RESUMEN}

El presente artículo lleva a cabo un análisis textual y temático de chistes étnicos contemporáneos en lengua rusa aparecidos en Internet entre 2010 y 2016. Estos textos tienen un denominador común: están protagonizados por georgianos. Durante el período soviético, únicamente un grupo selecto de nacionalidades protagonizaba los textos humorísticos étnicos y los georgianos pertenecían a él. El chiste étnico atribuía a estas nacionalidades unas características físicas, discursivas e idiosincráticas estables y estereotipadas, que explotaba para provocar la hilaridad en el oyente. Con el derrumbe de la Unión Soviética y el cambio de contexto político, económico y cultural, el chiste étnico en lengua rusa sufre importantes modificaciones en su estructura y temática. Asimismo, a partir de los años noventa del siglo XX, los chistes dejan de transmitirse de forma exclusivamente oral: la libertad de prensa permite su publicación en papel y pueden publicarse también en Internet. Los personajes no escapan a estos cambios: decrece la relevancia de sus características propias; los rasgos discursivos y las peculiaridades de comportamiento no son ya determinantes en el efecto cómico del chiste. El georgiano sigue apareciendo con frecuencia en los chistes étnicos por tradición y porque permite la elaboración de un concepto positivo del yo ruso.

PALABRAS CLAVE: Rusia, humor étnico, chiste étnico, estereotipo, georgiano

\section{TRENDS IN ETHNIC HUMOUR: AN ANALYSIS OF CONTEMPORARY JOKES IN RUSSIAN}

\section{ABSTRACT}

This article analyses both textually and thematically contemporary ethnic jokes in Russian, which were published in the Internet in the years 2010 to 2016. All the jokes share the commonality of having a Georgian as a main character. In Soviet times, only a few nationalities appeared as a main character in ethnic humorous texts and Georgians belonged to this select group. In ethnic jokes, certain stable, highly stereotyped characteristics - physical, discursive and idiosyncratic - were ascribed to a nationality, so that Russian-speakers easily recognised it. Moreover, ethnic jokes made use of these particularities to attain a comical effect. After the collapse of the Soviet Union and related political, economic and cultural transformations, ethnic jokes in Russian were affected by significant structural and thematic changes. Likewise, from the 1990's onwards, jokes were not only orally transmitted. Press freedom allowed printing and jokes started being published in the Internet. Characters are also affected: the relevance of their characteristics decrease; discourse features and behaviour particularities are not decisive for the comical effect. Georgians still appear as a main character in jokes as a part of the traditional also because in contemporary Russian ethnic jokes the Georgian character allows a development of a positive self-image.

KEYWORDS: Russia, ethnic humour, ethnic joke, stereotype, Georgian 
En una sociedad pluricultural donde domina la diversidad, las relaciones interétnicas son frecuentes y se manifiestan de formas muy diversos. El intercambio lingüístico constituye una de las más habituales. En muchas ocasiones, este intercambio no implica un diálogo; ocurre con frecuencia que uno de los grupos habla o escribe sobre los otros sin hacerlos partícipes, sin concederles el papel de interlocutores y la posibilidad de responder. Así sucede con los chistes. En este artículo analizaremos las manifestaciones lingüísticas que se producen en lengua rusa acerca de los georgianos en forma de chistes étnicos y que circulan en la Red.

A fin de llevar a cabo este análisis, se ha elaborado un corpus compuesto de cincuenta chistes étnicos sobre georgianos. Los textos se han extraído del sitio web Anekdot.ru, donde fueron publicados entre los años 2010 y 2016. Anekdot.ru, en funcionamiento desde 1995, publica diariamente chistes en lengua rusa. La utilización de este recurso como fuente para la elaboración del corpus responde a la inmensa popularidad de que goza Anekdot.ru en Runet, acrónimo por el que se conoce la comunidad rusohablante de Internet.

Según Mijaíl Alekséievski (2010: 5), ${ }^{1}$ «Anekdot.ru [es] el recurso más popular dedicado a este género. [...] en marzo de 1997 [...] los resultados estadísticos revelan que Anekdot.ru es el sitio más visitado de Runet». ${ }^{2}$ Si bien es cierto que la popularidad de este web declinó a finales de los años noventa, a fecha de 2010 y según este mismo especialista, seguía recibiendo más de 20.000 visitas diarias y a día de hoy sigue actualizándose de manera constante. Los chistes aparecidos en Anekdot.ru son, por tanto, actuales, significativos y representativos en el momento de su publicación y, por ende, consideramos que válidos para nuestro análisis. Con todo, es necesario precisar aquí que la fecha de publicación de un chiste no debe confundirse con la de su creación; los chistes son textos cuya datación resulta compleja. Así, «el texto del chiste varía, no está atado a un autor y su localización cronológica entraña una gran dificultad» (Melnichenko 2014: 30).

El chiste en lengua rusa recibe el nombre de anekdot. Como indica Melnichenko (2014), esta palabra proviene del griego anekdotos y significa «historia inédita o privada». En su origen, se empleaba para designar un rumor o cotilleo, significado que conservó hasta mediados del XVII. El término llegó a Rusia a mediados del siglo XVIII. En esta época, hacía referencia a un género literario oral cultivado por clases ilustradas. Algo más tarde, entre los siglos XVIII y XIX, el anekdot tomó en Rusia el significado de «texto escrito de carácter biográfico».

A principios del siglo XX ya aparecen en Rusia chistes de carácter político con el significado contemporáneo de esta palabra: un texto oral breve con un final

\footnotetext{
1 Todas las palabras con redacción original en alfabeto cirílico han sido romanizadas según la propuesta de Sánchez Puig y Alonso Cecilia (2012).

2 Todas las traducciones de las citas y los chistes son del autor. (N. del A.)
} 
inesperado y gracioso. Los chistes políticos gozaron de gran popularidad en Rusia durante el siglo XX. Incluso en plena época estalinista, cuando estaba prohibida la más leve crítica al Gobierno, los rusos inventaban y contaban chistes políticos sobre Stalin y otros dirigentes, o sobre la situación del país. Debe tenerse en cuenta que en la URSS la narración de un chiste, en particular de carácter político por su carga crítica hacia el Gobierno, se interpretaba como un acto de disidencia. En la época estalinista, este acto podía acarrear graves consecuencias. Así lo expresa Arjípova (2015):

En 1934 [...] se empieza a arrestar a los llamados «cuentachistes». [...] Los «cuentachistes» eran arrestados no solo por contar chistes, sino también por recitar pareados o cantar canciones; por expresar cualquier manifestación de folclore, que primero fue considerado contrarrevolucionario y más tarde, antisoviético. [...] En 1937 había tantos «cuentachistes» en los campos de trabajo que se publicó una disposición especial que ordenaba hacer distinción entre los que contaban chistes contra Stalin y los que solo bromeaban o cantaban canciones.

Sin embargo, en el presente estudio no centraremos nuestro análisis en el humor político en ruso, sino en los chistes étnicos, muy populares también en Rusia pese a que no han recibido tanta atención por parte del mundo académico como los de índole política. A diferencia de los chistes políticos, estos textos humorísticos centran su atención en personajes no rusos como ucranios, georgianos o chukchis. Los chistes de carácter étnico adquirieron relevancia durante la Perestroika, cuando las cuestiones de identidad y nacionalidad empezaron a poder debatirse abiertamente en la Unión Soviética (Draitser 1998: 10). Del humor étnico en lengua rusa nos interesa destacar su gran capacidad para consolidar estereotipos alrededor del protagonista del chiste, de etnia no rusa.

Antes de llevar a cabo el análisis de estos textos humorísticos, detallaremos a continuación algunas peculiaridades del chiste en lengua rusa. En primer lugar, debe destacarse que, a diferencia de lo que sucede en otras lenguas, el chiste en lengua rusa es considerado como un género textual independiente. Así, Jímik (2002: 17) afirma que «únicamente en la cultura oral rusa el fenómeno que a continuación analizaremos [el anekdot] tiene una denominación especial que lo diferencia de conceptos parecidos [en otras culturas]». Shmeliov y Shmeliova (2002: 20) destacan las «peculiaridades lingüísticas únicas» del chiste en lengua rusa, que «en el sistema contemporáneo ruso de géneros discursivos ocupa un lugar especial».

Estos autores caracterizan el chiste como género discursivo de la siguiente manera: en primer lugar, subrayan que se trata de un género que se transmite, nunca se crea. Así, el narrador del chiste no se arroga su autoría, aunque lo haya inventado. Lo habitual es que finja haberlo escuchado en una ocasión anterior cuando lo narra. Asimismo, el narrador prevé que su audiencia desconoce el chiste que está explicando; si el público ya lo ha oído, se contraviene la 
convención y el acto de habla como tal no puede ejecutarse. En cuanto a su estructura, este género discursivo está construido de acuerdo con unas normas específicas. El anekdot como género textual tiene una forma estable y casi siempre se construye en dos partes: introducción - en la que se pone al oyente en contexto- y desenlace, que suele ser corto y paradójico, además de contener la sal del chiste. Además, en el chiste étnico contemporáneo en lengua rusa figuran como protagonistas representantes de un reducido número de nacionalidades, que cuentan con características discursivas y de comportamiento estables y que son conocidos por todos los hablantes de lengua rusa. Por último, el chiste se compone de dos capas lingüísticas. Por un lado, las palabras del narrador, quien pone en juego una serie de estrategias narrativas al contar el chiste, puesto que está actuando ante una audiencia; por otro, el discurso de los personajes, que contiene elementos que los caracterizan.

Asimismo, resulta interesante destacar que el chiste ruso es el resultado de la interacción entre capas sociales y culturales distintas; en origen, el anekdot es un producto de la influencia de la cultura clásica europea en las clases educadas de Rusia. Durante el período soviético, que es el momento en que el chiste en lengua rusa toma su forma actual, el autor anónimo de muchos chistes es la inteliguentsia. Sin embargo, es el interés de la cultura popular por el género la que permite su difusión a gran escala primero en la Rusia Zarista y, más tarde, en la URSS.

Por supuesto, estamos tratando con una concepción clásica del anekdot, centrada en su realización oral en el contexto soviético. Del chiste en forma escrita se afirma que no respeta las convenciones. Se pone incluso en tela de juicio que deban ser llamados chistes los textos que se recogen en antologías o por Internet (Jímik 2002). Sin embargo, nosotros creemos que ha de tenerse en consideración que, a partir de los años noventa, tienen lugar en Rusia dos fenómenos que cambian la manera de entender el chiste en lengua rusa. En primer lugar, se consolida la libertad de prensa, que ya había hecho su tímida aparición en los ochenta. Los chistes, por tanto, dejan de ser actos clandestinos de disidencia y toman el ámbito público (Graham 2003). Precisamente uno de los medios más eficaces para su divulgación es publicarlos en papel; la oralidad ya no es el único medio de transmisión. En segundo lugar, Rusia accede a Internet. Shmeliov y Shmeliova (2005: 3) también registran estos cambios: «[el chiste] se transforma en un género literario y teatral (publicación de infinidad de antologías de chistes, abundancia de programas de radio y televisión en los cuales actores cuentan chistes), así como el género de la comunicación por internet (en sitios web tales como el mencionado Anekdot.ru).» Todo ello provoca necesariamente transformaciones en el formato del chiste.

La función del chiste en lengua rusa, por tanto, ha sufrido una transformación: tras la caída de la Unión Soviética nació un nuevo chiste, que se expresa por otras vías y que, naturalmente, modifica forma y contenido para adaptarse a los nuevos tiempos. Sin embargo, hasta el momento hemos citado 
cambios estructurales en el anekdot; las transformaciones políticas y sociales que sacudieron a la URSS en los ochenta y a la Federación de Rusia en los noventa influyeron también en el tratamiento que el chiste dispensa a los personajes protagonistas.

Antes de considerar estos cambios, resulta imprescindible entender de qué manera se presenta tradicionalmente a los georgianos en el humor étnico soviético. Recordemos que la tipología de personajes que aparecen en estos chistes es limitada, y el oriundo de Georgia pertenece a este grupo selecto (Shmeliov y Shmeliova 2002: 50). El georgiano es, por tanto, uno de los personajes prototípicos de los chistes étnicos en lengua rusa del siglo XX. Los georgianos en estos chistes se presentan de forma estereotipada y no necesitan de presentación ya que, «son tan famosos como los personajes de cuentos infantiles» (Shmeliov y Shmeliova 2002: 50).

En los chistes étnicos de los años ochenta y noventa, los georgianos son fácilmente reconocibles, puesto que se les atribuyen con regularidad unos rasgos determinados. Tanto Draitser (1998) como Shmeliov y Shmeliova (2002), tras analizar diferentes chistes étnicos sobre georgianos, coinciden en el hecho de que estos textos transmiten una imagen fuertemente estereotipada. Enumeramos a continuación las características más importantes del georgiano según Shmeliov y Shmeliova (2002: 50-52) acompañados de chistes a modo ilustrativo. ${ }^{3}$

1. EL GEORGIANO ES UN INDIVIDUO QUE VISTE ROPA CARA, PERO EN EXCESO LLAMATIVA, DE MAL GUSTO INCLUSO.

Chiste:

Un georgiano habla con un amigo sobre la ropa que llevará en la próxima cita:

- Me pondré unos zapatos blanquísimos, unos pantalones blancos-blancos y una camisa más que blanca.

El amigo le dice:

-Ponte también una gorra verde y parecerás una botella de leche.

\section{AL GEORGIANO LE ENCANTA OSTENTAR Y HACER GALA DE SU RIQUEZA, SEA REAL O FICTICIA.}

Chiste:

Centro de Moscú, mediodía, 32 grados a la sombra. Por una avenida incandescente va un Volga $^{4}$ con cristales tintados. Dentro, cuatro georgianos con abrigos y sombreros de piel.

Al fin, uno de ellos no aguanta más y dice con voz quejumbrosa:

- ¡Venga, vamos a abrir por lo menos una ventana!

Los otros le responden:

$-{ }_{i} \mathrm{No}$, que todos se piensen que tenemos aire acondicionado!

\footnotetext{
3 Todos los chistes citados a provienen del corpus.

${ }^{4}$ Automóvil soviético de gama alta.
} 
3. EL GEORGIANO EN LOS CHISTES DE LENGUA RUSA ES UNA PERSONA HOSPITALARIA, QUE AMA LAS REUNIONES, LA SOBREMESA Y LOS BRINDIS; ES GENEROSO, A VECES EN EXCESO.

Chiste:

Tras cenar en un restaurante, dos georgianos pelean por ver quién deja la propina más generosa a la encargada del guardarropa. Uno coge el abrigo y dice:

-Toma, mil dólares de propina.

El segundo dice:

-Toma, mil dólares y quédate el abrigo.

\section{EN SU RELACIÓN CON LAS MUJERES, EL GEORGIANO TRATA A LOS MIEMBROS DEL SEXO OPUESTO} COMO SERES INFERIORES.

Chiste:

Un georgiano se encuentra con otro, que va acompañado de una hermosa mujer. El primero empieza a hablar con el segundo de la belleza de su acompañante, de quien dice numerosos cumplidos. La mujer, por fin, se siente obligada a agradecer las palabras del amigo, quien, al oírla, responde:

-Tú, mujer, a callar cuando los hombres hablan.

5. EL PODER SOVIÉTICO MOLESTA A LOS GEORGIANOS, EN TANTO QUE LES IMPIDE DISFRUTAR DE LA VIDA.

Chiste:

Un georgiano es aceptado en el PCUS. Le comunican que, ahora que es miembro del Partido, no debe ni beber vino, ni ganar mucho dinero, ni cortejar a mujeres. Tras aleccionarlo, le preguntan:

- ¿Está usted dispuesto a dar la vida por los ideales del comunismo?

A lo que el georgiano responde:

- Por supuesto, ipara qué quiero yo una vida así!

En su análisis, Draitser (1998) hace hincapié en que en la época soviética nació la idea de que el georgiano es rico y tiene facilidad para ganar dinero. Esta caracterización contrasta con la del ruso, humilde y sencillo. Los años inmediatos a la caída del comunismo trajeron estrecheces económicas para gran parte de la población de la ex URSS. En este contexto, el contraste de la pobreza rusa con la pretendida riqueza georgiana se exagera. En el análisis de este autor, se caracteriza al personaje georgiano de la siguiente manera:

1. EL GEORGIANO SABE CÓMO HACER FORTUNA RÁPIDAMENTE, AL CONTRARIO QUE LOS RUSOS. CON TODO, ESTE DINERO ABUNDANTE TIENE UN ORIGEN OSCURO.

Chiste:

Un georgiano conduce un destartalado Moskvich. ${ }^{5}$ Un flamante Volga lo adelanta con facilidad y se para en medio de la carretera, bloqueando el paso. De él sale un georgiano vestido a la última moda y le dice:

- ¿Por qué avergüenzas a tu pueblo? ¡Mírame! Hace justo un año que salí de la cárcel y ya tengo un Volga.

\footnotetext{
${ }^{5}$ Automóvil soviético de gama baja.
} 
Regresa al coche, da un portazo y se va.

El georgiano del Moskvich aprieta los dientes y sigue su camino. Algo más tarde un lujoso Mercedes lo adelanta y se para en medio de la carretera, bloqueando el paso. Un georgiano, con ropa aún más lujosa, sale del coche y dice:

- ¿Es que no tienes vergüenza? Ni medio año hace que dejé el talego y ya estoy al volante de un Mercedes.

Cierra y se marcha.

El georgiano masculla entre dientes:

- ¡Hace un año que salí! ¡Medio año hace que salí! ¿Es que no pueden darme tiempo a llegar a casa el día que me han soltado?

\section{AL GEORGIANO LE SOBRA EL DINERO Y PUEDE ADQUIRIR CON FACILIDAD PRODUCTOS QUE EN LOS AÑOS OCHENTA DEL SIGLO PASADO ERAN INALCANZABLES PARA UN RUSO.}

Chiste:

En una iglesia un georgiano le pide a Dios dinero para comprar un coche. Junto a él, un ruso reza por un litro de vodka. Al final, el georgiano se cansa, le da al ruso diez rublos y le dice:

- Toma, cómprate una botella y no molestes a Dios con tus tonterías.

3. POR SUPUESTO, ESTA ABUNDANCIA DE DINERO DE DUDOSA LEGALIDAD TIENE UN PODEROSO EFECTO CORRUPTOR.

Chiste:

Aeropuerto de Tbilisi. Un georgiano hace cola para comprar un billete de avión. Cuando llega su turno, pone mil rublos en el pasaporte y dice en la ventanilla:

- ¡Querido! Un billete a Moscú.

- No quedan - le responden desde la ventanilla.

El pasajero añade mil rublos.

- ¡Querido! Deme un billete a Moscú. ¡Tengo mucha prisa!

El agente coge el teléfono y dice:

-Operadora, póngame con la fiscalía.

El georgiano no puede creer que un paisano lo esté denunciando. Pero oye como el agente dice:

-Señor Fiscal, se ha cancelado su reserva. No podrá volar a Moscú.

\section{SE PRESENTA AL GEORGIANO COMO UN HOMBRE EXTREMADAMENTE VIRIL, OBSESIONADO POR EL}

SEXO.

Un georgiano va al médico.

-Doctor, ¿qué puedo hacer? Me he quedado impotente.

- Hábleme un poco de su vida sexual.

-Es de lo más normal: cada noche lo hago con mi mujer; cada tarde, con mi amante; durante la jornada laboral, tengo a mi secretaria. Y eso es todo.

-Está claro, todo es fruto de su hiperactividad sexual. Con un estilo de vida así, era inevitable que desarrollara la impotencia.

$-¿$ Es por eso?

- Por supuesto.

- Gracias a Dios. Pensaba que era culpa de la masturbación. 
5. ESTA MASCULINIDAD SE PRESENTA EN OCASIONES COMO DESARROLLADA EN EXCESO. ASÍ, EL GEORGIANO NO ES ÚNICAMENTE UN MUJERIEGO, SINO TAMBIÉN UN PERVERTIDO.

Chiste:

El georgiano a los miembros del jurado:

- Yo pensaba que «Por la seducción de un menor» era un brindis y no un artículo del código penal.

Entre estos análisis aparecen algunas divergencias, pero también puntos coincidentes: en primer lugar, se presenta al georgiano como un personaje fuertemente estereotipado, dotado con unos rasgos estables y definidos. En segundo lugar, tanto Draitser como Shmeliov y Shmeliova destacan la importancia de la imitación del acento en la caracterización del georgiano protagonista del anekdot. Cuando cuenta un chiste, el narrador debe emular el habla del georgiano, definida por un acento característico y por su desconocimiento de la lengua rusa, que, por tanto, habla con errores. La lengua rusa empleada por el personaje georgiano se aleja significativamente de la norma culta. Esta impostación constituye un recurso muy efectivo para provocar la hilaridad en el oyente, empleado en el humor étnico de muchas otras lenguas. En este chiste se observa con claridad de qué modo se explota este recurso.

\section{Chiste:}

Una escuela en Georgia. Lección de lengua rusa. La maestra pide a los alumnos que hagan el análisis sintáctico de la oración «Los georgianos van a lavarse a la sauna». El estudiante Goguia responde:

-Los georgianos... Sustantivo. Van a lavarse... Preposición. A la sauna... Pronombre.

Por supuesto, en el humor étnico en lengua rusa el georgiano no es el único personaje que comete incorrecciones lingüísticas. También los ucranios o los chukchis hablan un ruso incorrecto. Esta caracterización lingüística permite equiparar al protagonista del chiste con el provincianismo y, por ende, adjudicarle el rasgo de la ignorancia, dado que el desconocimiento de la lengua se puede asociar fácilmente a una baja inteligencia.

En los chistes escritos, por supuesto, es imposible conseguir este efecto propio de la oralidad. Con todo, existen estrategias para llevar a cabo esta realización oral del acento: se modifica la grafía correcta de determinadas palabras a fin de reproducir el modo en que el georgiano pronuncia algunos motes. Huelga decir que el acento, que el narrador imita en el momento de contar un chiste étnico sobre georgianos, difiere en gran medida del acento real de un georgiano al hablar en ruso. Se trata únicamente de una máscara lingüística que produce un efecto cómico y forma parte también de la caracterización prototípica del personaje. En palabras de Shmeliov y Shmeliova (2002: 41): «Es de una importancia extrema la función caracterológica que tienen las características discursivas del personaje. Sucede que las peculiaridades que caracterizan el discurso del personaje se corresponden con los rasgos de su carácter.» 
Sean rasgos positivos o negativos, idiosincráticos o lingüísticos, el georgiano es un personaje prototípico, cuya personalidad está sólidamente elaborada. Los chistes étnicos son determinantes a la hora de generar estos estereotipos alrededor de una nacionalidad. Con todo, su función no termina ahí, puesto que tan importante como la creación del estereotipo es su consolidación; la narración y trasmisión de estos chistes es de suma importancia para que el estereotipo se actualice y sobreviva en el tiempo.

De los chistes sobre georgianos podemos deducir que los rusos tienen de Georgia y sus gentes una imagen estable y elaborada. Aquí es necesario recordar que los georgianos no son simplemente ciudadanos de otro país, sino miembros de una nación que ha mantenido fuertes vínculos históricos Rusia. Georgianos y rusos han convivido durante mucho tiempo bajo un solo estado De hecho, en los chistes sobre georgianos $-\mathrm{y}$ otras nacionalidades que aparecen con frecuencia en los chistes étnicos en lengua rusa como ucranios y estonios - estos personajes no son considerados como un extranjero, sino como un inorodets. Este término hace referencia a "pueblos que viven codo con codo con los rusos, pero que no dominan el ruso y se diferencian de los rusos por determinados rasgos de carácter y conducta» (Shmeliov y Shmeliova 2002: 71). Asimismo, que el hecho de que un personaje venga caracterizado por una máscara lingüística - lo cual, como hemos visto, sucede en el caso de los georgianos - determina que el personaje del chiste es un inorodets y no un extranjero (Shmeliov y Shmeliova 2002: 71).

Los lazos históricos que unen a Georgia y Rusia pueden explicar este alto grado de significatividad del georgiano en el humor étnico en ruso. Ambos países han mantenido estrechos contactos a lo largo de la historia. Georgia entró en la esfera de influencia rusa a finales del siglo XVIII. En 1921, tras un breve período de independencia que siguió a la Revolución de 1917 y el desmembramiento del Imperio zarista, el país caucásico pasó a formar parte del nuevo estado bolchevique. Si bien con el desmembramiento de la URSS Georgia y la Federación de Rusia se transformaron en países independientes, el país caucásico no se convirtió simplemente en el extranjero; este estado forma parte ahora del «extranjero cercano».

En el caso del humor étnico, es de especial interés el fenómeno que tiene lugar tras la formación de la URSS. Georgia - junto con otros territorios que habían constituido el Imperio ruso - pasó a formar parte del recién creado estado comunista, teóricamente en pie de igualdad con el resto de nacionalidades que conformaban el nuevo país. Sin embargo, en el proyecto soviético de encaje de la realidad multiétnica no se intentaba conseguir la plena asimilación de los nuevos ciudadanos. La política de nacionalidades oficial de la URSS se caracterizaba por el internacionalismo.

Por un lado, en el marco de este paradigma internacionalista, se desarrolla la idea de que la Unión Soviética está constituida por diferentes naciones, que deben convivir armónicamente para crear un estado multinacional que supere 
las diferencias de origen. La realización a escala individual de este proyecto es el homo sovieticus, un nuevo individuo que encarna la superación de las barreras nacionales. Por otro lado, sin embargo, dado que se trata de una unión voluntaria de naciones, se fomenta la conciencia nacional de los pueblos que la constituyen. Incluso comunidades que en el momento de la formación de la URSS no habían desarrollado plenamente su identidad nacional, son dotadas ahora de una lengua, una historia y una conciencia (Lurié 2011: 148), en base al concepto primordialista que de la identidad étnica se tenía en la Unión Soviética (Dadúiev 2013: 169).

Es posible relacionar la tendencia a la consolidación estereotípica del chiste étnico en lengua rusa con la política soviética de relaciones interétnicas, pues se otorgaban, de manera artificial e inalterable, unas características prototípicas a los diferentes pueblos que integraban la URSS. Con todo, el chiste étnico soviético no es únicamente un resultado de esta política, sino también una reacción: la narración de chistes sobre otras nacionalidades de la Unión Soviética constituía, al igual que la narración de chistes de índole política, un acto de disidencia, ya que las críticas a la política de nacionalidades oficial estaban prohibidas. En la época soviética era casi imposible publicar chistes étnicos puesto que «era muy fácil ver en ellos un menosprecio al origen nacional, lo que en la mentalidad soviética se consideraba sinónimo de crimen» (Burykin 2002: 64).

Como puede verse, las características del chiste étnico guardan una íntima relación con el contexto histórico en que se desarrolla. Con la desaparición de la Unión Soviética, el chiste étnico ha de modificarse también. Hemos mencionado con anterioridad que durante los años noventa se consolidan dos fenómenos - la libertad de prensa y la aparición de nuevos medios de comunicación- que tendrán una influencia considerable en los aspectos estructurales del chiste en lengua rusa. La nueva realidad deja sentir también sus efectos negativos sobre el chiste. Graham (2003) anuncia la decadencia general del género. Por su parte, Shmeliov y Shmeliova (2005: 292) constatan con las siguientes palabras esta pérdida de importancia del chiste.

\footnotetext{
En los últimos años ha disminuido notablemente el «peso específico» del género discursivo del chiste en la comunicación diaria. Como antes, siguen apareciendo nuevos chistes, pero parece que los rusohablantes se cuentan chistes con mucha menos frecuencia; gradualmente se pierde la necesidad de narrar a los amigos el último chiste escuchado.
}

Los chistes étnicos en lengua rusa sobre georgianos no escapan a esta tendencia: entre el 8 de noviembre de 1995 - fecha de creación de Anekdot.ruy el 31 de diciembre de 2016, se publicaron en esta página un total de 488 chistes sobre georgianos. Solamente 59 chistes, es decir algo más de 12\% del total, aparecieron entre los años 2010 y 2016. Esta reducción del número de chistes en este período puede explicarse por la tendencia general al decaimiento del género que hemos mencionado previamente. 
Sin embargo, consideramos que los cambios que afectan al chiste étnico sobre georgianos no son únicamente cuantitativos: el análisis de estos chistes, aparecidos en Anekdot.ru en los últimos seis años, aporta información interesante sobre los cambios que se producen en el estereotipo del georgiano. Es necesario observar aquí que el estereotipo es un constructo dinámico que toma elementos de la tradición para generar o modificar nuevos estereotipos (Diátlov, 2010: 144). Así, es natural que muchos de los chistes que aparecen en el período de estudio escogido sean chistes antiguos o tomen elementos que ya existían en chistes precedentes. Estos textos no son innovadores; se caracterizan por actualizar el estereotipo del georgiano descrito anteriormente en este artículo. Así, en estos chistes se sigue presentando al georgiano como un machista:

Chiste:

En un banquete, un georgiano levanta su copa y hace un brindis:

- Me gustaría brindar por las mujeres, que, sin nosotros los hombres, no pueden vivir, del mismo modo que un pez no puede vivir sin agua.

La mujer sentada a su lado dice:

- ¿De qué está hablando, joven? Hace veinte años que vivo sin mi marido y me encuentro perfectamente. No me considero infeliz en absoluto.

El georgiano responde:

-Señora, propongo un brindis por el pescado fresco.

Asimismo, el personaje georgiano de los chistes publicados recientemente sigue teniendo problemas con la lengua rusa:

Un georgiano entra en una tienda. Quiere comprar una gallina. Ve unos huevos y, como no sabe decir «gallina» en ruso, dice:

- ¡Quiero comprar a su mamá!

Además, su dinero sigue teniendo un poder corruptor:

Un georgiano le dice a su hijo:

-Escucha, hijo, que te voy a contar un cuento para que te duermas. Había una vez un topo que compró un carné de conducir...

- ¡Papá! ¡Que los topos son ciegos!

-Hijo, escucha, tú te piensas que en Moscú venden carnés pero no venden certificados médicos, ¿verdad?

Como podemos ver en el chiste que citamos a continuación, el georgiano se vuelve a presentar, de manera estereotipada, como un personaje adinerado, capaz de adquirir un avión valorado en dos millones de rublos. El chiste pertenece, como se explicita en la introducción, a una época pasada, cuando se creía que los georgianos gozaban de un elevado poder adquisitivo que contrastaba con la precariedad económica de los rusos.

Chiste:

Chiste de mediados de los años ochenta, cuando un salario eran 180 rublos.

Un georgiano se sienta en el avión y le pregunta a la azafata: 
- ¿Cuánto cuesta este avión?

La azafata le responde:

-Dos millones de rublos.

Y el georgiano dice:

$-¡ A y$, ay! Dos años de trabajo.

Sin embargo, hay también chistes nuevos e incluso fenómenos llamativos, como por ejemplo el de la reelaboración. Estas reelaboraciones toman como base un chiste antiguo, pero, a diferencia de los ejemplos que acabamos de mencionar, modifican el final con un añadido de creación reciente. El chiste que citamos a continuación es, como se indica de nuevo en la introducción, un chiste clásico soviético. Hemos destacado el añadido, actual, en letra cursiva.

Chiste:

Antiguo chiste soviético. Ser georgiano es un título; ser judío es una vocación; ser gitano es una profesión y ser ruso es un destino.

Todos dan limosna a los gitanos, pero nadie los quiere.

Los judíos lo consiguen todo por sí mismos, y nadie los quiere.

Los georgianos no necesitan nada; en su tierra crece de todo. Con más razón, nadie los quiere.

Los rusos dan limosna a todos y por eso nadie los quiere.

Es interesante observar que este chiste en concreto ha sido reelaborado para mejorar la imagen de los rusos. Recordemos aquí que en muchas ocasiones afirma que el propósito del humor nacional es la elaboración de estándares de identidad nacional, tanto en clave positiva -identificación positiva con el grupo nacional - como negativa: yo y los que no son rusos (Dombróvskaia 2014). En el contexto de la política étnica soviética no se podía, de manera oficial, atacar a otras nacionalidades. Con la desaparición de este marco de relaciones interétnicas, el humor queda liberado de restricciones y se instrumentaliza.

En el período 2010-2016 se pueden encontrar chistes de nueva creación con protagonista georgiano en los que, a diferencia de lo que hemos visto hasta este momento, no se lleva a cabo una actualización del estereotipo, pero sí se trabaja en la imagen positiva del ruso. El anekdot, lo hemos visto con anterioridad, es fruto de un contexto histórico concreto y refleja la realidad del momento en que aparece. Así, abundan en esta etapa contemporánea chistes que hacen referencia a las relaciones entre Rusia y Georgia. Cabe recordar aquí que estas relaciones han venido marcadas en los últimos años por una gran tensión política que, en ocasiones, como en el año 2008, degeneraron en conflictos armados entre ambos países. De esta situación nacen chistes como el que mencionamos acto seguido.

Chiste:

Los georgianos han construido una máquina de guerra terrestre única en el mundo entero. Se llama Lazika. En cuarta, es capaz de alcanzar los $60 \mathrm{~km} / \mathrm{h}$. Y marcha atrás, jllega a los 120 ! 
De nuevo, además de hacer referencia a la situación de conflicto bélico entre Rusia y Georgia, este chiste proyecta una imagen positiva de los rusos: en este caso, los presenta vencedores de una guerra en la que los georgianos huyen despavoridos. A diferencia de los años que siguieron al derrumbe de la URSS, cuando el ejército de la Federación de Rusia tenía serios problemas para terminar con éxito empresas bélicas, ahora vence con facilidad. Se han conjurado en parte los traumas provocados por las derrotas bélicas en Chechenia y que, según Laruelle (2011: 65) marcaron profundamente la conciencia colectiva rusa en los años noventa del siglo XX. En otros contextos, encontramos también chistes en el que se produce un enfrentamiento de carácter no militar con un georgiano, del que el ruso sale vencedor.

En el compartimento de un tren hay una chica que lleva medias con bordado. Frente a ella se sienta un georgiano, que le pregunta:

- ¿A dónde llevan esos bordados?

La chica, juguetona, le responde:

- A la estación.

- ¿Si te doy 100 rublos me enseñas esa estación? - replica el georgiano.

El marido baja de un salto de la litera de arriba.

-Mira, hijo de $\mathrm{p}^{* * *}$, dame 200 rublos y te enseño al jefe de la estación.

Asimismo, en este contexto de antagonismo, en 2015 sucede un hecho que genera multitud de chistes sobre georgianos: a principios de ese año Mijaíl Saakashvili, presidente de Georgia en la época del conflicto armado con Rusia, es nombrado gobernador de la región ucrania de Odesa. No es difícil establecer una clara conexión entre Ucrania y Georgia, pues comparten muchas similitudes a ojos rusos: ambas repúblicas eran parte constituyente de la URSS y se apresuraron para proclamar su independencia tras el colapso de la Unión; para la política rusa, ambos estados forman parte del «extranjero cercano»; georgianos y ucranios son personajes fuertemente estereotipados en el chiste étnico en lengua rusa; ambos países han mantenido en los últimos años tensas relaciones políticas con Rusia, marcadas en ocasiones por el conflicto armado, que se ha resuelto con victorias para los rusos en forma de cesiones territoriales.

En los chistes que citamos a continuación no encontramos al georgiano protagonista del chiste étnico en lengua rusa, con unos rasgos de carácter y conducta definidos y fuertemente estereotipados. Los protagonistas son, en realidad, dos países. Bajo una óptica rusa, Ucrania, cuya política se caracteriza por la inestabilidad, el rechazo a lo ruso, y las tendencias pro occidentales, vive una situación tan desesperada que recurre a los servicios de políticos extranjeros como Saakashvili, pro occidental e igualmente denostado también por la opinión pública rusa. Hay una equiparación entre Georgia y Ucrania, ambos presentados como países inmersos en situaciones problemáticas. Rusia, por el contrario, parece estar alejado de las turbulencias que sacuden a los vecinos. 
Chiste:

Me ha llamado un amigo de Tbilisi. Dice que da miedo andar por la calle: cogen a los georgianos por la calle y los envían a Ucrania para que hagan de ministros.

Chiste:

El presidente de Ucrania Poroshenko consulta a Occidente. Quiere saber quién puede salvar la economía de su país. Los expertos occidentales le responden:

- Es una tarea propia de un kamikaze.

«Comprendo la indirecta», piensa Poroshenko. Les da la siguiente orden a sus servidores:

-Dicen que en Georgia hay un tipo que hace milagros, un tal Kamikaze. ${ }^{6}$ ¡Es el único que nos puede salvar! ¡Encontradlo enseguida y traedlo!

Al cabo de un tiempo regresan e informan al presidente:

- No hemos encontrado a Kamikaze. Nos hemos traído a unos cuantos georgianos huérfanos. Y a Saakashvili, que se ha apuntado.

Chiste:

Malo es aquel georgiano que no sueña con llegar a ministro en Ucrania.

Por último, hallamos chistes protagonizados por georgianos en los que las características prototípicas del personaje - su manera de comportarse, su acento o sus rasgos físicos - no son relevantes para conseguir el efecto hilarante. En estos textos, se podría cambiar la nacionalidad del personaje principal y el resultado sería idéntico.

Chiste:

Un georgiano le habla a su suegra:

-Mamá, ¿esto qué es? De desayunar, mijo; de comer, mijo; de cenar, mijo. ¿Qué se piensa que soy? ¿Un pájaro?

Chiste:

Un georgiano va a una casa de citas. Entra en una habitación y ve a una chica muy delgada.

Le pregunta:

-Oye, ¿cuánto pesas?

-42 kilos - le responde la chica.

$-¿$ Puedes andar?

-Sí.

- Pues sal de aquí.

Chiste:

Un georgiano le pregunta a su vecino ruso:

-Oye, querido, ¿dónde te has puesto tan moreno?

- El domingo estuve trabajando en el huerto.

- Uy, querido, ven mañana a broncearte a mi casa.

En estos chistes se incluye el personaje étnico, pero no hay actualización de ninguna de sus características. Es cierto que en los textos originales se reproduce

${ }^{6}$ En ruso, la pronunciación de esta palabra es kamikadze. La terminación -adze es típica de muchos apellidos georgianos. 
la máscara lingüística del personaje georgiano. Sin embargo, su pronunciación o sus errores lingüísticos no son las causas del efecto humorístico. Tampoco los rasgos idiosincráticos del personaje constituyen la sal del chiste. El anekdot emplea otros recursos. Con todo, se incluye a un personaje georgiano. ¿Por qué? Recordemos que los georgianos son una de las pocas nacionalidades que protagonizan los chistes étnicos en lengua rusa. Su presencia refuerza la comicidad en el texto, aunque su aparición no sea determinante.

\section{BIBLIOGRAFÍA}

ALEKSÉIEVSKI, M. (2010), «Anekdoty ot Ziugánova: folklor v sovreménnoi politícheskoi borbé» [Los chistes de Ziugánov: el folklore en la lucha política contemporánea], en Antropologuícheski fórum, 12, 1-36.

ARjÍPOVA, A. (2015), Vzaimodéistvie vlasti i folklora [Interacción entre poder y folklore] [en línea], en Moscú: Postnauka, 22 marzo 2015 [Consulta: 2 febrero 2017]. Disponible en: <http://postnauka.ru/video/44274>

BURYKIN, A. (2002), "Anekdoty o chukchaj kak sotsiokulturnoe yavlenie (zametki spetsialista)» [Los chistes sobre chukchis como fenómeno sociocultural (apuntes de un especialista)], en El chiste como fenómeno cultural. Mesa redonda, San Petersburgo.

DADÚIEV, M. (2013), «Kontseptualnye osnovy etnopolitiki v sovreménnoi Rossi» [Fundamentos conceptuales de la política étnica en la Rusia contemporánea], en Politícheskie Nauki, 2, 169-173.

DOMBRÓVSKAIA, I. (2014), Yumor v kontekste razvitia [El humor en el contexto del desarrollo], Montreal, Accent Graphics Communications.

DraITSER, E. (1998), Taking Penguins to the Movies: Ethnic Humor in Russia, Detroit, Wayne State University Press.

GRAHAM, S. (2003), A Cultural Analysis of the Russo-Soviet Anekdot, Pittsburgh, Universidad de Pittsburgh.

JÍMIK, V. (2002), «Anekdot kak unikálnoe yavlenie russkoi rechevói kultury» [El chiste como un fenómeno único en la cultura oral rusa], en El chiste como fenómeno cultural. Mesa redonda, San Petersburgo.

LARUELLE, M. (2011), «Nacionalismo y construcción estatal en Rusia: Un consenso social debilitado», Revista CIDOB d'afers internacionals, 96, 63-79.

LURIÉ, S. (2011), «'Druzhba naródov’ v SSSR: natsionalny proekt ili primer spontánnoi mezhetnícheskoi samoorganizatsi?» [La «Amistad de los pueblos» en la URSS: ¿proyecto nacional o ejemplo de autoorganización interétnica espontánea?], en Obschestvénnye nauki i sovreménnost, 4, 145-156.

MELNICHENKO, M. (2014), Sovetski anekdot: ukazatel siuzhetov [El chiste soviético: índice de materias], Moscú, Novoe Literaturnoe Obozrenie.

SÁnChez Puig, M.; Alonso CeCILIA, C. (2012), Diccionario temático Ruso-español, Españolruso, Madrid, Ediciones Hispano Eslavas.

SHMELIOV, A. y SHMELIOVA, E. (2002), Russki anekdot: tekst i rechevoi zhanr. [El chiste ruso: texto y género discursivo], Moscú, Yazyki Slaviánskoi Kultury. 
SHMELIOV, A. y SHMELIOVA, E. (2005), «Russki anekdot v dvadtsat pervom veke: transformatsi rechevogo zhanra» [El chiste ruso en el siglo veintiuno: transformaciones del género discursivo], en Zhanr i kontsept, 4, 292-298.

SHMELIOV, A. y SHMELIOVA, E. (2014), «Etnícheskie stereotipy v russkij anekdotaj» [Estereotipos étnicos en los chistes rusos], en Otéchestvennie zapiski, 4(61), 70-77. 